\title{
PET-guided repeat transsphenoidal surgery for previously deemed unresectable lateral disease in acromegaly
}

\author{
Waiel A. Bashari, MSc, MRCP(UK), ${ }^{1,2}$ Russell Senanayake, MSc, MRCP(UK), ${ }^{1,2}$ \\ Olympia Koulouri, PhD, MRCP(UK),, ${ }^{1,2}$ Daniel Gillett, MSc, ${ }^{1,3}$ James MacFarlane, MRCP(UK), \\ Andrew S. Powlson, MRCP(UK), ${ }^{1,2}$ Antia Fernandez-Pombo, MD, ${ }^{1,2}$ Gul Bano, FRCP, ${ }^{7}$ \\ Andrew J. Martin, FRCS, ${ }^{8}$ Daniel Scoffings, FRCR, ${ }^{1,4}$ Heok Cheow, FRCR, ${ }^{1,3,4}$ \\ losif Mendichovszky, PhD, FRCR, ${ }^{1,3,4}$ James Tysome, PhD, FRCS, ${ }^{1,5}$ Neil Donnelly, MSc, FRCS, ${ }^{1,5}$ \\ Thomas Santarius, PhD, FRCS, ${ }^{1,6}$ Angelos Kolias, PhD, FRCS, ${ }^{1,6}$ Richard Mannion, PhD, FRCS, ${ }^{1,6}$ \\ and Mark Gurnell, PhD, FRCP1,2
}

${ }^{1}$ Cambridge Endocrine Molecular Imaging Group, ${ }^{2}$ Metabolic Research Laboratories, Wellcome Trust-MRC Institute of Metabolic Science; Departments of ${ }^{3}$ Nuclear Medicine, ${ }^{4}$ Radiology, ${ }^{5}$ Otolaryngology, and ${ }^{6}$ Neurosurgery, University of Cambridge and National Institute for Health Research Cambridge Biomedical Research Centre, Addenbrooke's Hospital, Hills Road, Cambridge; and Departments of ${ }^{7}$ Endocrinology and ${ }^{8}$ Neurosurgery, St George's University Hospital, London, United Kingdom

OBJECTIVE The object of this study was to determine if revision transsphenoidal surgery (TSS), guided by ${ }^{11} \mathrm{C}$ methionine PET/CT coregistered with volumetric MRI (Met-PET/MR ${ }^{C R}$ ), can lead to remission in patients with persistent acromegaly due to a postoperative lateral disease remnant.

METHODS The authors identified 9 patients with persistent acromegaly following primary intervention (TSS \pm medical therapy \pm radiotherapy) in whom further surgery had initially been discounted because of equivocal MRI findings with suspected lateral sellar and/or parasellar disease (cases with clear Knosp grade 4 disease were excluded). All patients underwent Met-PET/MR ${ }^{C R}$. Scan findings were used by the pituitary multidisciplinary team to inform decision-making regarding repeat surgery. Revision TSS was performed with wide lateral exploration as guided by the PET findings. Endocrine reassessment was performed at $6-10$ weeks after surgery, with longitudinal follow-up thereafter.

RESULTS Met-PET/MRCR revealed focal tracer uptake in the lateral sellar and/or parasellar region(s) in all 9 patients, which correlated with sites of suspected residual tumor on volumetric MRI. At surgery, tumor was identified and resected in 5 patients, although histological analysis confirmed somatotroph tumor in only 4 cases. In the other 4 patients, no definite tumor was seen, but equivocal tissue was removed. Despite the uncertainty at surgery, all patients showed immediate significant improvements in clinical and biochemical parameters. In the 8 patients for whom long-term follow-up data were available, insulin-like growth factor 1 (IGF-1) was $\leq 1.2$ times the upper limit of normal (ULN) in all subjects and $\leq 1$ times the ULN in 6 subjects, and these findings have been maintained for up to 28 months (median 8 months, mean 13 months) with no requirement for adjunctive medical therapy or radiotherapy. No patient suffered any additional pituitary deficit or other complication of surgery.

CONCLUSIONS This study provides proof of concept that Met-PET/MRCR can be helpful in the evaluation of residual lateral sellar/parasellar disease in persistent acromegaly and facilitate targeted revision TSS in a subgroup of patients. https://thejns.org/doi/abs/10.3171/2020.3.FOCUS2052

KEYWORDS acromegaly; lateral parasellar; Met-PET/MRCR; extended TSS

ABBREVIATIONS DA = dopamine agonist; FSPGR = fast spoiled gradient-recalled echo; GH = growth hormone; IGF-1 = insulin-like growth factor 1; MDT = multidisciplinary team; Met-PET = PET using ${ }^{11} \mathrm{C}$-methionine; Met-PET/MRCR = Met-PET coregistered with volumetric MRI; $\mathrm{PA}=$ pituitary adenoma; $\mathrm{RT}=$ radiotherapy; $\mathrm{SSA}=$ somatostatin analog; TSS = transsphenoidal surgery; ULN = upper limit of normal.

SUBMITTED February 1, 2020. ACCEPTED March 3, 2020.

INCLUDE WHEN CITING DOI: 10.3171/2020.3.FOCUS2052. 
A CROMEGALY is a systemic disorder characterized by growth hormone $(\mathrm{GH})$ and insulin-like growth factor 1 (IGF-1) hypersecretion and is almost always caused by a $\mathrm{GH}$-secreting pituitary adenoma (PA). ${ }^{16}$ The excess morbidity and mortality associated with active acromegaly can be mitigated or reversed by treatments that restore $\mathrm{GH}$ and IGF-1 levels to normal, with a recent study suggesting that normalization of IGF-1 is of particular importance. ${ }^{23}$

In most specialist pituitary centers, transsphenoidal surgery (TSS) remains the primary treatment for the majority of GH-secreting PAs ${ }^{13,19}$ and is cost-effective as compared with other treatment modalities. ${ }^{14}$ However, even in the hands of experienced pituitary neurosurgeons, most microscopic and endoscopic case series report remission rates $>85 \%$ following primary surgery for microadenomas but just $40 \%-60 \%$ for macroadenomas. ${ }^{7,8,13}$ The likelihood of achieving complete resection with full biochemical remission has been linked to tumor invasiveness and proximity to adjacent critical neurovascular structures. ${ }^{17}$ Parasellar disease is currently classified using the Knosp grading system, 9,17 in which cross-sectional imaging demonstrating a grade 2 tumor (between the intercarotid line and the lateral tangent) or higher (grade 3 or 4) may be perceived as inoperable (comparable to grades D and E in the earlier Hardy classification). ${ }^{6,17}$

For patients with residual disease following primary pituitary surgery, treatment options include medical therapy and/or radiotherapy (RT). ${ }^{8}$ The former typically includes one or more of somatostatin analog (SSA), GH receptor antagonist, or dopamine agonist (DA) therapy. While these treatments are highly effective in delivering symptom relief and biochemical control and can ameliorate acromegaly-related comorbidities, they often require longterm (even lifelong) administration, which comes at significant financial cost (it has been estimated that the lifetime cost for pharmacotherapy in acromegaly is between 41 and 59 times that of surgery, depending on the drug). ${ }^{14}$ For those receiving $\mathrm{RT}$, the beneficial effects can take several years to be fully realized, ${ }^{5}$ and medical treatment must be continued during the intervening period. Moreover, new pituitary hormone deficits occur in up to $50 \%$ of patients within 5-10 years after RT, 3,8,14 and concerns remain over the potential increased risk of cerebrovascular disease and second tumors. ${ }^{18}$

An important factor limiting the role for, and the efficacy of, revision surgery in functioning pituitary tumors is the ability of current cross-sectional imaging techniques to reliably identify sites of low-volume residual or recurrent disease and to discriminate disease from posttreatment change. ${ }^{1}$ In acromegaly, more accurate assessment of the true extent of cavernous sinus involvement may allow reconsideration of endoscopic TSS in selected cases or help inform RT planning (e.g., when considering stereotactic radiosurgery).

PAs are characterized by high amino acid synthesis, and PET using ${ }^{11} \mathrm{C}$-methionine (Met-PET) has therefore been proposed as an adjunctive imaging strategy for localization of residual or recurrent tumor that is not readily visualized on MRI alone. ${ }^{1}$ When coregistered with volumetric (i.e., thin slice) MRI (Met-PET/MR ${ }^{\mathrm{CR}}$ ), Met-PET has been shown to help identify the location and extent of residual tumor in patients with persistent acromegaly following primary therapy and can therefore inform decision-making regarding adjunctive therapy.,11,20

Considering these initial encouraging findings, we wished to determine whether Met-PET/MR ${ }^{\mathrm{CR}}$ could specifically facilitate revision TSS in patients with persistent acromegaly following primary therapy, but in whom repeat surgery had initially been discounted because of equivocal MRI findings and a strong suspicion of lateral sellar and/or parasellar disease.

\section{Methods \\ Patients}

Patients with residual active acromegaly following primary surgery and referred to our center for consideration for Met-PET/MR ${ }^{\mathrm{CR}}$ in the period 2017-2019 were considered eligible for inclusion in the case series if revision surgery had been initially discounted by a United Kingdom specialist pituitary multidisciplinary team (MDT) because of equivocal postoperative MRI findings and a suspicion of lateral sellar/parasellar disease. Patients in whom there was a reasonable suspicion of Knosp grade 4 disease were not included (as surgery would not be considered an option in this context). All patients had biochemical confirmation of active acromegaly (persistently raised IGF-1 > 1.5 times the upper limit of normal [ULN], and $\mathrm{GH}$ nadir $>0.4 \mu \mathrm{g} / \mathrm{L}$ following a 75 -gm oral glucose tolerance test). Nine consecutive patients were identified and the findings in these cases are reported here. The study received institutional approval, and all patients provided written informed consent for PET-guided revision surgery.

\section{Biochemical Measurements}

$\mathrm{GH}$ and IGF-1 levels were measured in a Clinical Pathology Accreditation Ltd. laboratory (CPA) with relevant internal and external quality assurance as required by the CPA. Serum GH concentration was measured using a solid-phase, two-site, time-resolved fluorometric assay (DELFIA, PerkinElmer Life and Analytical Sciences Inc.) calibrated to IS 98/574 (analytical sensitivity 0.01 $\mathrm{ng} / \mathrm{ml}$; intraassay coefficient of variation $<5 \%$ across the range $0.025-25 \mathrm{ng} / \mathrm{ml})$. Serum IGF-1 was measured using a solid-phase, enzyme-labeled, chemiluminescent immunometric assay (Siemens Immulite 2000, Siemens Medical Solutions Diagnostics Ltd.) calibrated to IS $87 / 518$ (analytical sensitivity $20 \mathrm{ng} / \mathrm{ml}$; intraassay coefficient of variation $<10 \%$ across the range $25-1600 \mathrm{ng} / \mathrm{ml}$ ), and results are reported as values times the ULN.

\section{Radiopharmaceutical Synthesis and Scan Protocol}

All patients fasted for 4 hours before PET/CT scanning. They were injected with Met (dose range 250-410 MBq) and scanned using a Discovery 690 PET/CT scanner (GE Healthcare), as previously described..$^{11,12}$ The uptake time for $\mathrm{PET} / \mathrm{CT}$ was standardized at 20 minutes. In patients who were receiving medical therapy at the time of referral, treatment withdrawal was undertaken for $>3$ months for SSA and $>1$ month for DA therapy. Met-PET and fast spoiled gradient-recalled echo (FSPGR) MRI were coreg- 
istered as previously reported, ${ }^{11,12}$ and findings were verified using the free open-source software 3D Slicer. Images were reviewed by the Cambridge specialist pituitary MDT, comprising pituitary neurosurgery and otolaryngology, endocrinology, neuroradiology, neuro-oncology, neuropathology, and neuro-ophthalmology.

\section{Transsphenoidal Surgery}

Following review of the PET images by the specialist MDT, a consensus was reached that redo TSS for lateral sellar/parasellar disease could be offered. The patients were reviewed in the joint pituitary (neurosurgery/ endocrinology) clinic, where the images and all available treatment options (including continued medical management, RT/radiosurgery, or redo surgery) were discussed. All patients decided to proceed with PET-guided revision TSS, which was jointly conducted by a neurosurgeon and an otolaryngologist. Following induction of general anesthesia, patients were placed supine on the operating table, with the trunk raised $10^{\circ}$ and the head neutral with $10^{\circ}$ rotation toward the surgeon. A horseshoe headrest was used to position the head. A binostril technique was routinely used, with two surgeons working together after the nasal stage of the procedure. Intraoperative image guidance was used as necessary (e.g., for patients with poorly pneumatized sphenoid sinus). Met-PET/MR ${ }^{\mathrm{CR}}$ was used preoperatively to determine whether the residual tumor was resectable and to target the surgery relative to other anatomical structures, in particular, the carotid artery. The findings from the preoperative Met-PET/MR ${ }^{\mathrm{CR}}$ study were displayed in the operating room but were not incorporated in neuronavigation images. The sella was opened with a combination of microdrills and punches. In the cases described here, particular care was taken to extend the opening laterally toward the cavernous sinus on the side identified by the preoperative PET. This lateral extension of the sellar opening, which is a few millimeters more than the standard TS approach, is a key part of the technique employed in such cases. This typically requires exposure of the parasellar internal carotid artery (ICA), which was done using a diamond burr and micro-Doppler. After opening the dura, with the use of micro-Doppler as necessary, extensive exploration of the lateral sellar and parasellar areas was performed with the use of $0^{\circ} / 30^{\circ}$ endoscopes and ring curettes; samples were taken, and abnormal tissue was removed. In those cases in which no abnormal tissue was seen, exploration of the whole fossa was performed, and samples were taken for subsequent histological assessment as indicated. Sellar repair was performed with a nasoseptal flap if intraoperative CSF leakage was encountered. In the postoperative period, patients were monitored on a neurosurgical ward and were discharged home when stable from the neurological and endocrinological perspectives.

\section{Statistics}

Descriptive analysis was undertaken.

\section{Results}

Nine consecutive patients were included in the series
( 3 women, 6 men; mean age 43 years, range 27-65 years). The primary intervention had been TSS in all patients (once in cases 2, 3, 4, 7, 8, and 9; twice in cases 1, 5, and 6). Adjunctive postoperative medical therapy included SSA (all patients except in case 5) and DA (cases 3 and 6); RT, after the first of two TS procedures, was performed in only case 1 and predated the PET-directed surgery by $>$ 10 years (Table 1$)$.

\section{Imaging}

Clinical MRI findings both at initial presentation to us, following previous treatment (surgery, medical therapy, RT), and after PET-guided revision TSS are described in Table 1. In all 9 patients, Met-PET/MR ${ }^{\mathrm{CR}}$ revealed lateral sellar/parasellar tracer uptake at the site of suspected tumor remnants identified on volumetric MRI (Figs. 1-4). In addition, tracer uptake was also seen (as expected) at sites of residual normal pituitary gland: coregistration of the PET/CT and volumetric MRI allowed accurate localization of sites of Met uptake, which was essential for differentiating uptake by normal functioning pituitary tissue from uptake by residual adenoma.

\section{Surgical Findings}

All operations were uneventful, and no complications were encountered. Intraoperatively, abnormal tissue consistent with tumor was evident in 5 patients (cases 1, 4, 7, $8,9)$. Grossly abnormal tissue was not identified intraoperatively in the other 4 patients (cases 2, 3,5,6), but we proceeded to thorough exploration and took specimens from areas that were thought to contain equivocal tissue, focusing on the side identified as abnormal by the preoperative PET scan. Brisk bleeding from the medial wall of the cavernous sinus was encountered in only 1 patient (case 7), which may have indicated perforation of the medial wall of the cavernous sinus in this case. In none of the other patients was the medial wall of the cavernous sinus seen to be clearly breached.

\section{Clinical Outcomes}

Acromegaly-related symptoms and signs fully resolved in 8 of 9 patients following PET-guided TSS (Table 1). The patient in case 2, despite rapid biochemical normalization, continued to experience clinically significant chronic headache that did not change following TSS. Her headache was only responsive to treatment with thrice daily short-acting octreotide, and this was restarted at 8 weeks after her operation, precluding any further biochemical follow-up. Assessment of vision did not reveal any new deficits after PET-guided TSS in any patient.

\section{Biochemical Outcomes}

Early postoperative endocrine assessment, performed at $6-10$ weeks postsurgery and at least 12 weeks after medical therapy was discontinued, confirmed a marked improvement in biochemical control in all patients, with only 1 patient returning to IGF-1 > 1.2 times the ULN (Table 1 and Fig. 5). Importantly, there were no new pituitary deficits following surgery. Long-term maintenance of remission was assessed by serial IGF-1 measurements 


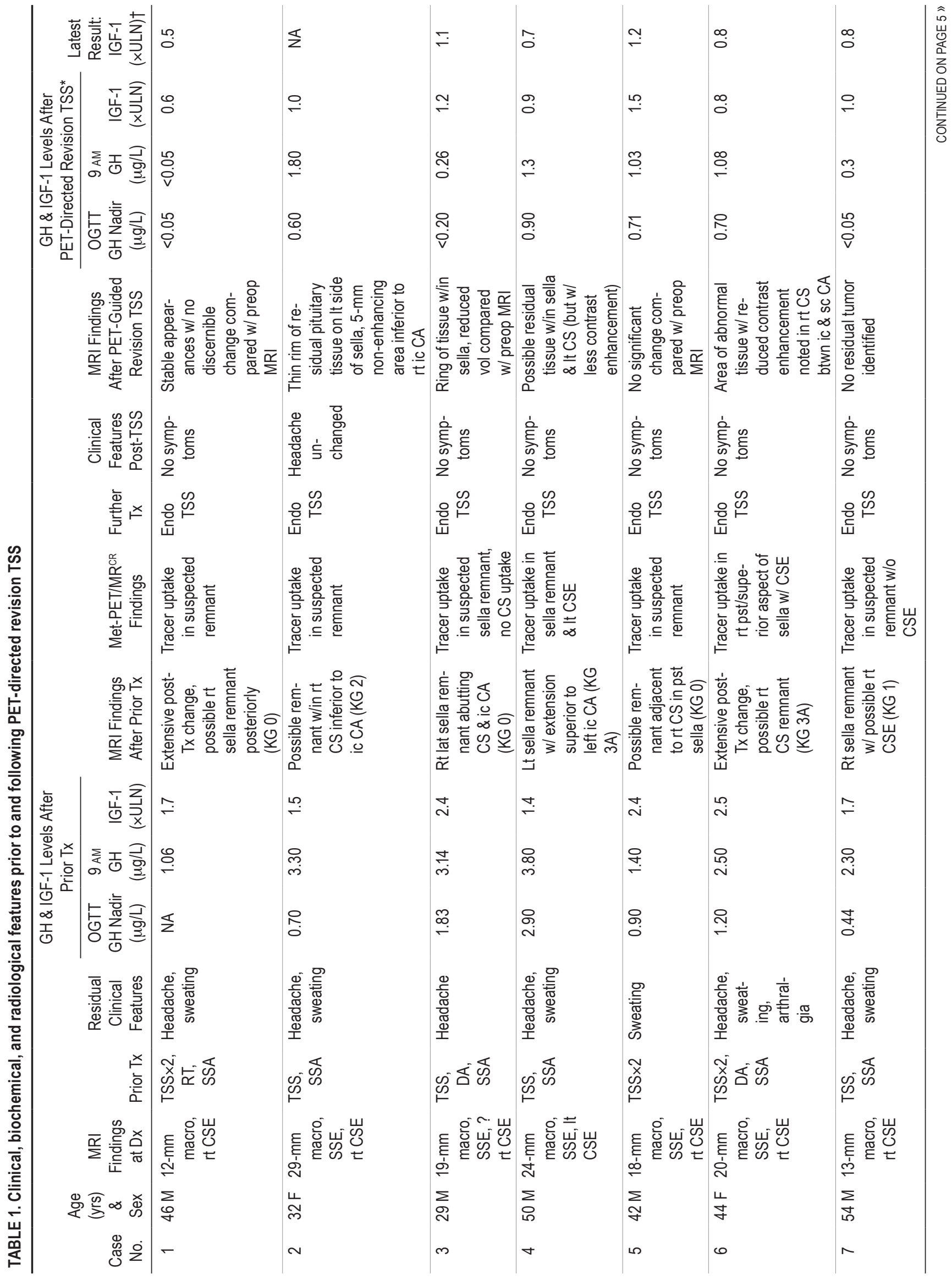




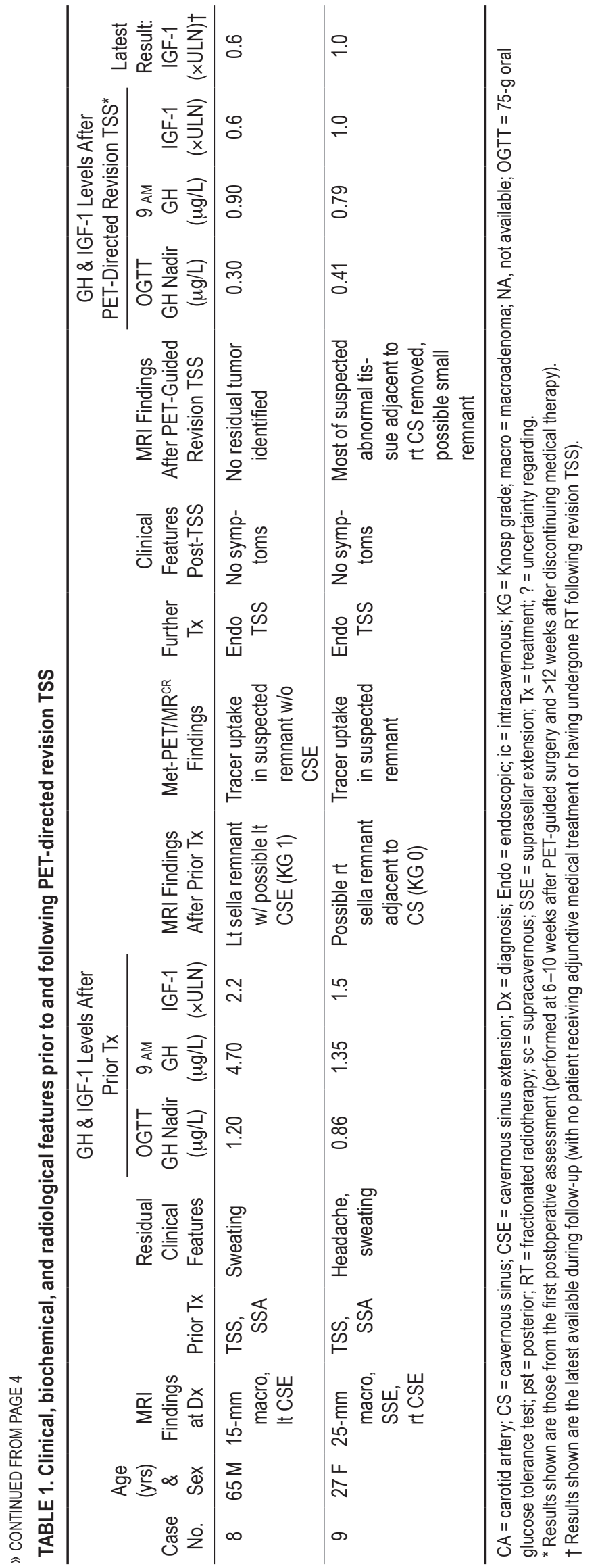

in 8 patients (excluding the patient in case 2 , as outlined previously). In all of these patients, the initial response was sustained (IGF- $1 \leq 1$ times the ULN in 6 cases and $\leq$ 1.2 times the ULN in 2 cases at last review), with a median duration of follow-up of 8 months (mean 13 months, range 4-28 months). None of these 8 patients received adjunctive medical therapy or (further) RT.

\section{Histology and Immunohistochemistry}

Of the 5 patients (cases $1,4,7,8,9$ ) who had abnormal tissue identified intraoperatively, 4 (cases $1,4,8,9$ ) had histological and immunohistochemistry (IHC) confirmation of a somatotroph adenoma. Of the 4 patients who had equivocal areas sampled without any grossly abnormal tissue, 1 patient (case 6) had histological and IHC confirmation of the presence of a somatotroph adenoma.

\section{Discussion}

In this proof-of-concept study, we have shown that Met$\mathrm{PET} / \mathrm{MR}^{\mathrm{CR}}$ can help guide revision TSS to achieve clinical and biochemical remission, even in patients who have previously been deemed unsuitable for further surgical intervention by a specialist pituitary MDT (Table 1 and Figs. $1-5)$. These findings support earlier work ${ }^{11,20}$ and suggest that molecular endocrine (functional) imaging with radiotracers such as Met should be considered in patients who may be potential candidates for further definitive treatment (e.g., repeat TSS, stereotactic radiosurgery) but in whom conventional imaging is equivocal, and even when lateral parasellar disease is suspected.

A potential role for Met-PET in the evaluation of PAs was first recognized in the 1980s. ${ }^{2}$ Met has the advantage of readily crossing the blood-brain barrier and is taken up at a cellular level via specific amino acid transporters as a prerequisite for subsequent protein synthesis. Accordingly, Met is particularly suited for the assessment of all PA subtypes, as protein synthesis is a common shared feature (even in "clinically nonfunctioning" tumors). ${ }^{10-12,21}$

Met-PET has been specifically advanced as a tool for the assessment of residual pituitary tumor following TSS. Tang and colleagues, in a cohort of 33 postoperative cases, were able to successfully localize sites of residual tumor that were not evident on conventional MRI in 14 patients. $^{22}$ This cohort included 5 patients with GH excess, 2 of whom proceeded to Gamma Knife radiosurgery guided by Met-PET. In a retrospective study comparing Met-PET/CT with ${ }^{18} \mathrm{~F}-\mathrm{FDG} \mathrm{PET} / \mathrm{CT}$ and reporting findings in 43 patients (16 with acromegaly), Met-PET/CT was superior to ${ }^{18} \mathrm{~F}$-FDG PET/CT for tumor localization in all tumor subtypes. ${ }^{4}$ Rodriguez-Barcelo et al. compared the role of Met-PET/MR ${ }^{\mathrm{CR}}$ with that of ${ }^{18} \mathrm{~F}-\mathrm{FDG}-\mathrm{PET}$ in 17 patients with acromegaly, with 7 patients found to have residual active disease following surgery. ${ }^{20}$ Met-PET/MR ${ }^{\mathrm{CR}}$ was shown to have a sensitivity and specificity of $86 \%$, as compared to a sensitivity of $28 \%$ and specificity of $100 \%$ for ${ }^{18} \mathrm{~F}$-FDG PET/CT. We have also previously described a role for Met-PET/MR $\mathrm{MR}^{\mathrm{CR}}$ in the assessment of 26 patients with persistent acromegaly following previous treatment. Met-PET/MR ${ }^{\mathrm{CR}}$ identified the site of active residual disease in 25 cases $(96 \%)$ to facilitate PET-directed TSS in 14 

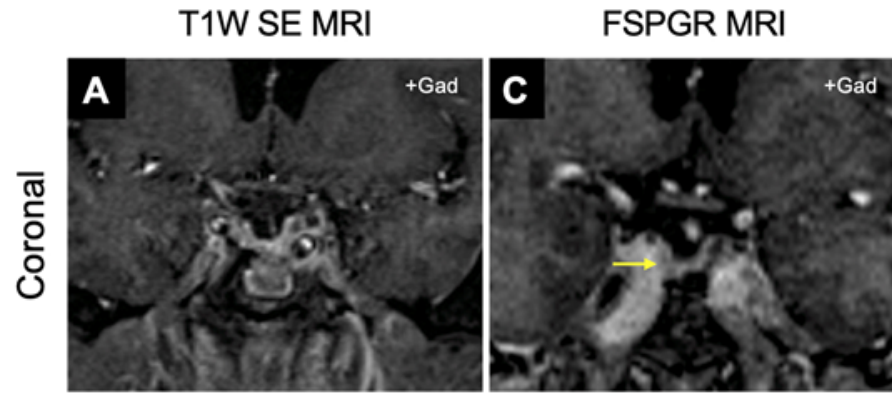

\section{Co-registered ${ }^{11} \mathrm{C}$ - methionine PET/CT \&} FSPGR MRI

\section{D-rendered model}
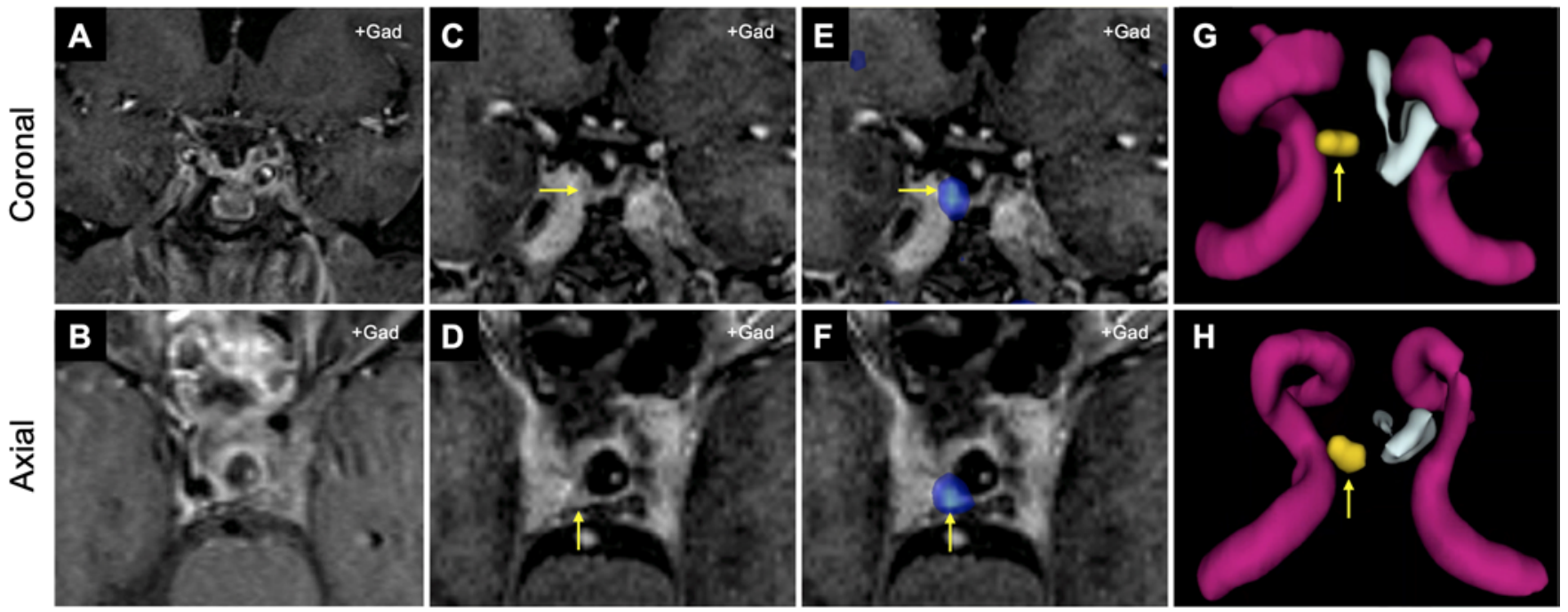

FIG. 1. Case 1. Images obtained in a 46-year-old man with persistent acromegaly who had undergone two previous TSSs, fractionated RT > 10 years earlier, and long-term SSA therapy. Coronal (A) and axial (B) T1-weighted (T1W) gadolinium-enhanced (+Gad) spin echo (SE) MRI showed extensive posttreatment changes, with left-sided infundibular deviation (the original pretreatment scan was not available but was reported to show a right-sided, 12-mm adenoma). Coronal (C) and axial (D) +Gad FSPGR MRI demonstrated a possible tumor remnant situated laterally and posteriorly on the right side (yellow arrows). Coronal (E) and axial (F) Met-PET/MR ${ }^{C R}$ demonstrated tracer uptake at the site of the suspected tumor remnant (yellow arrows). Coronal (G) and axial $(\mathrm{H})$ computerized 3D-rendered models of the coregistered study showed the location of the suspected tumor remnant (yellow arrows) and its proximity to the right carotid artery. Remaining normal pituitary tissue and infundibulum are shown in gray.

T1W SE MRI

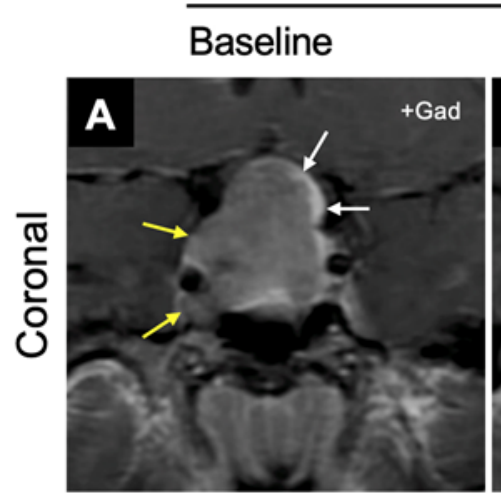

FIG. 2. Case 2. Images obtained in a 32-year-old woman with persistent acromegaly who had undergone previous TSS and SSA therapy. Coronal T1W +Gad SE MRI (A) from the initial presentation showed a large macroadenoma with suprasellar and right-sided cavernous sinus extension (yellow arrows); the normal gland is displaced to the left of the sella (white arrows). After the primary intervention, coronal T1W +Gad SE MRI (B) revealed a possible tumor remnant in the right parasellar region, inferior to the right intracavernous carotid artery (yellow arrow); the normal gland is visible on the left side of the sella (white arrow). Coronal (C) and axial (D) +Gad FSPGR MRI revealed similar appearances. Coronal (E) and axial (F) Met-PET MR ${ }^{\mathrm{CR}}$ confirmed the site of the suspected tumor remnant (yellow arrows) and the normal gland (white arrows). Nasopharyngeal uptake (red arrows) has been attenuated to improve visualization of the sella and cavernous sinuses.

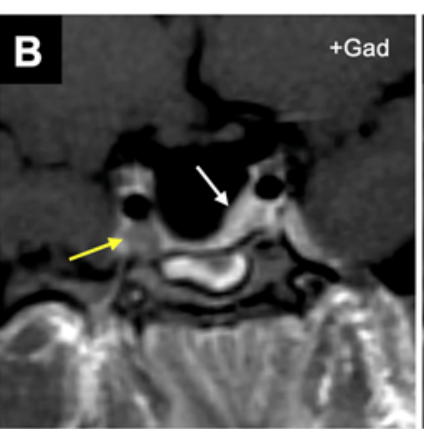

FSPGR MRI

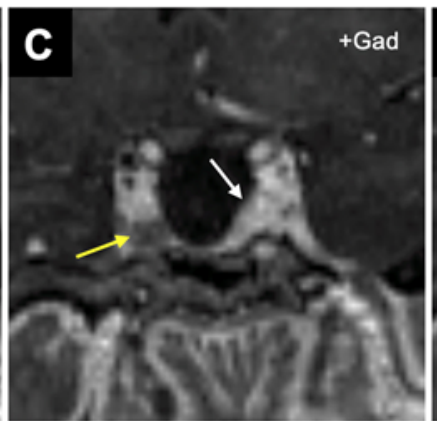

\section{Co-registered ${ }^{11} \mathrm{C}$ - methionine PET/CT \& FSPGR MRI}
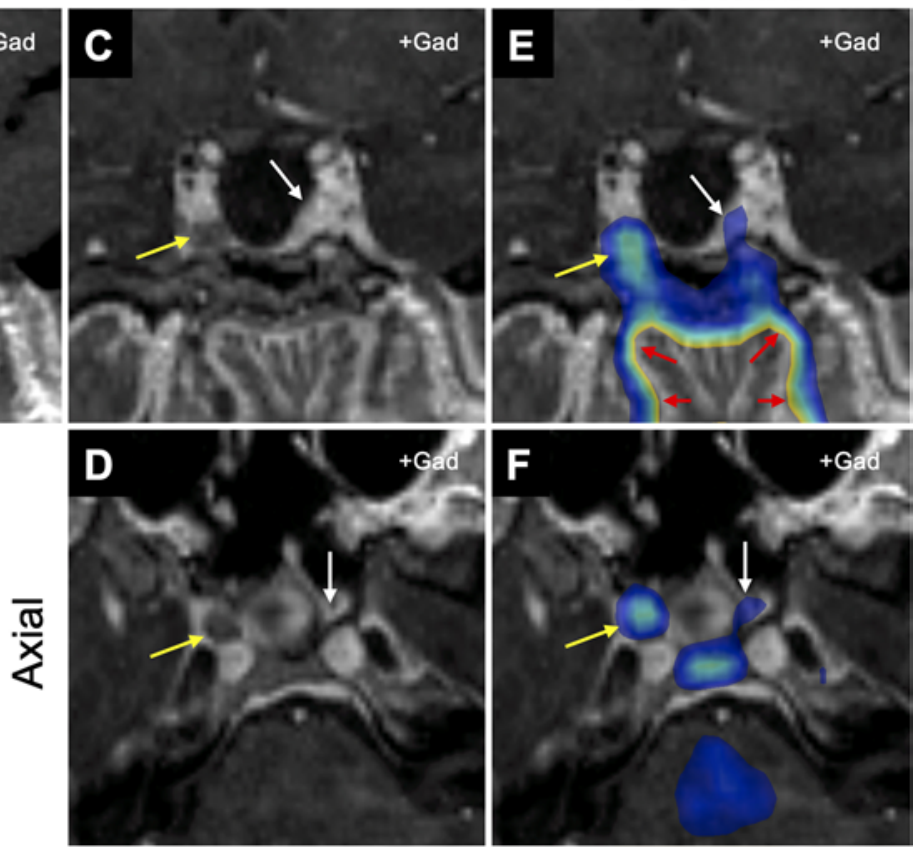
T1W SE MRI

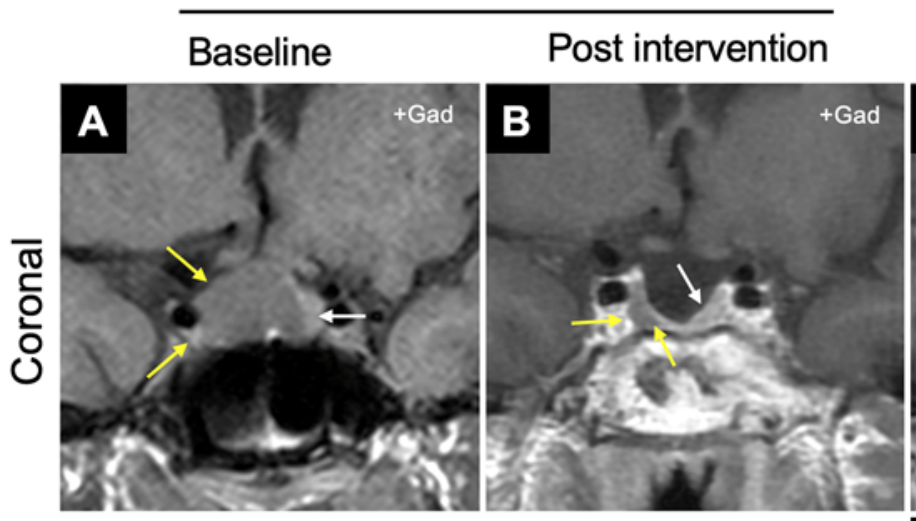

FIG. 3. Case 3. Images obtained in a 29 -year-old man with persistent acromegaly who had undergone previous TSS, SSA therapy, and DA therapy. Coronal T1W + Gad SE MRI (A) at the initial presentation showed a macroadenoma with suprasellar extension and encroachment on the right cavernous sinus (yellow arrows), with the normal gland displaced to the left (white arrow). Coronal T1W +Gad SE MRI (B) after the primary intervention showed a suspected tumor remnant adjacent to, and possibly encroaching, the right cavernous sinus (yellow arrows); the normal gland is situated on the left side of the sella (white arrow). Coronal (C) and axial (D) +Gad FSPGR MRI confirmed an area of abnormal signal laterally and posteriorly on the right (yellow arrows); the normal gland is located on the left (white arrow). Coronal (E) and axial (F) Met-PET/MR ${ }^{C R}$ confirmed tracer uptake at the site of the suspected tumor remnant (yellow arrows) and within the remaining normal gland (white arrows).
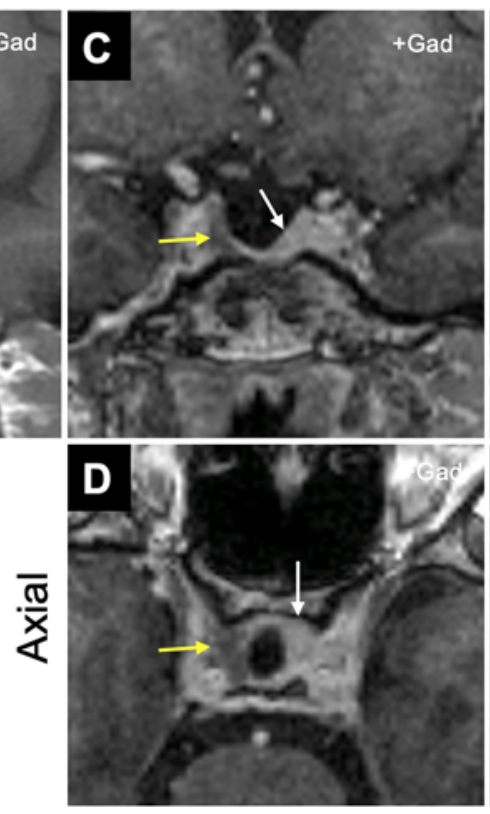
Co-registered ${ }^{11} \mathrm{C}$ - methionine PET/CT \& FSPGR MRI
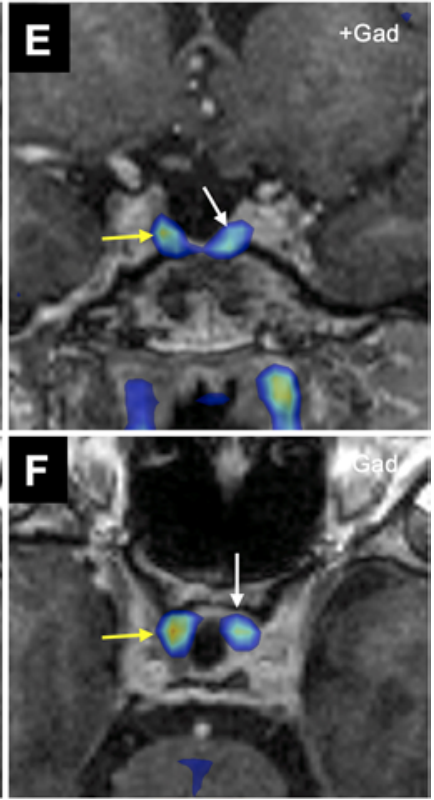

T1W SE MRI

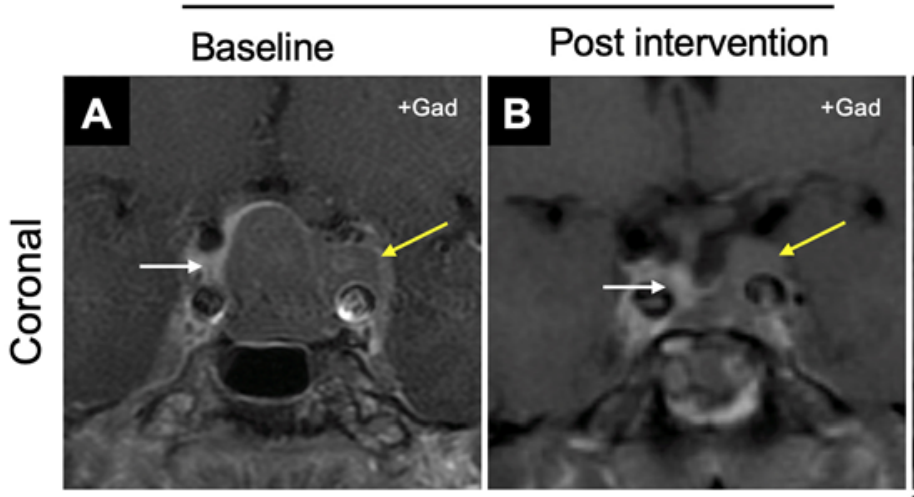

FIG. 4. Case 4. Images obtained in a 50-year-old man with persistent acromegaly who had undergone previous TSS and SSA therapy. Coronal T1W +Gad SE MRI (A) showed a large macroadenoma with suprasellar and left-sided cavernous sinus extension (Knosp grade $3 \mathrm{~A}$, yellow arrow); the normal gland is displaced to the right of the sella (white arrow). After the primary intervention, coronal T1W +Gad SE MRI (B) showed left sella and left cavernous sinus remnants (yellow arrows). Coronal (C) and axial (D) +Gad FSPGR MRI confirmed a left-sided tumor remnant with extension into the left cavernous sinus superiorly and posteriorly (yellow arrows) and normal gland on the right (white arrows). Coronal (E) and axial (F) Met-PET/MR ${ }^{C R}$ confirmed the site of the suspected tumor remnant (yellow arrows) and the remaining normal gland (white arrows).
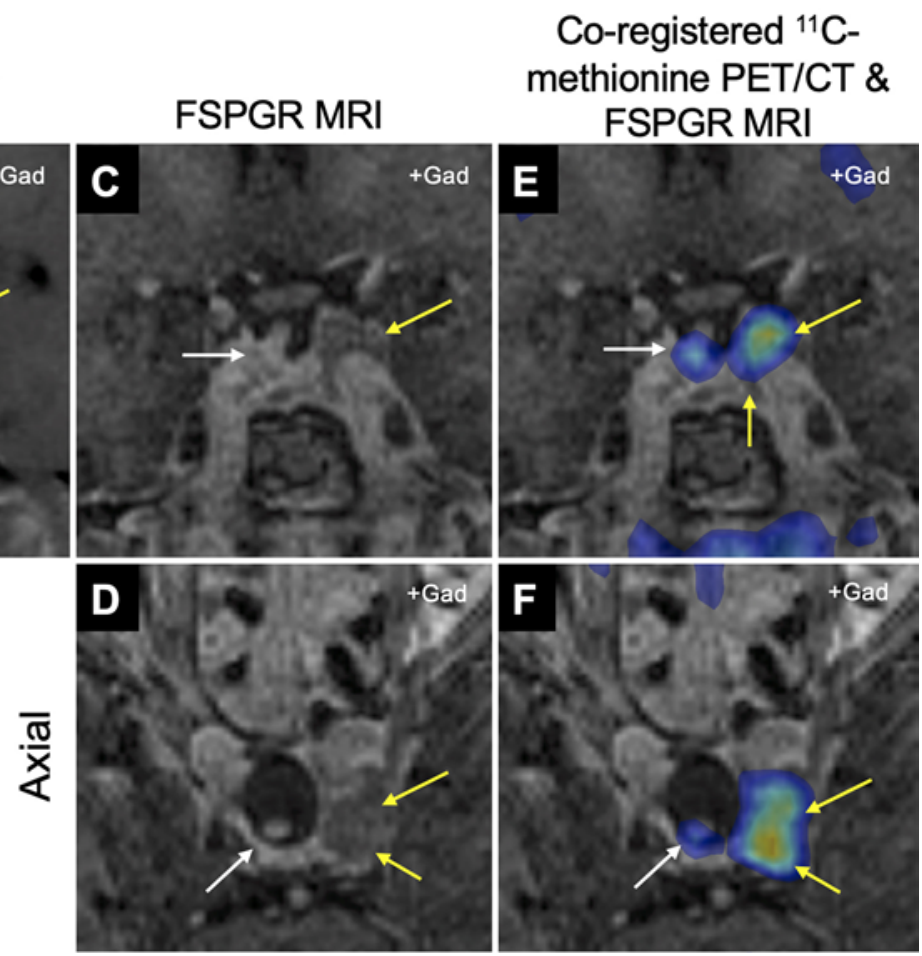


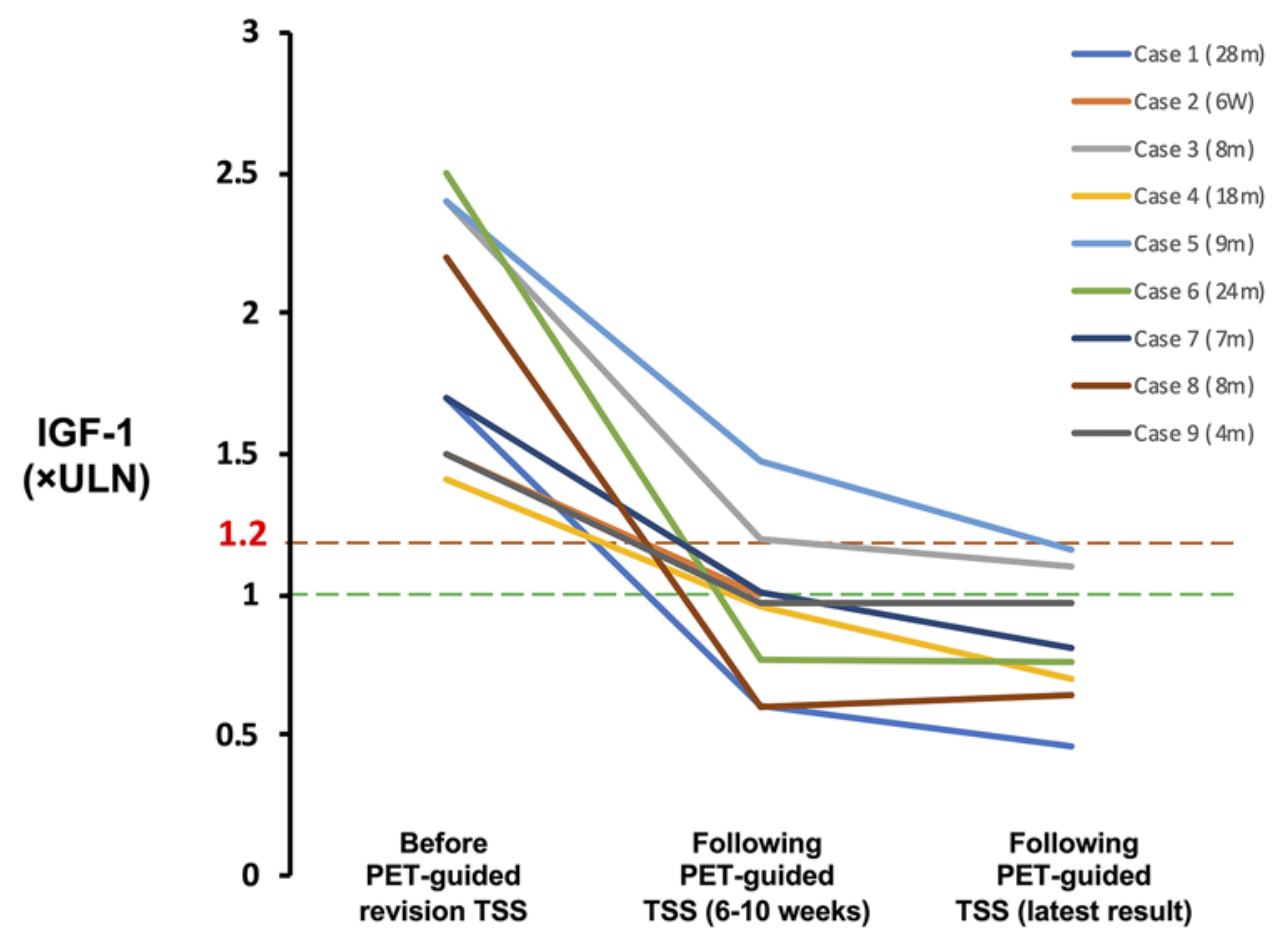

FIG. 5. Graph showing biochemical remission following PET-guided repeat TSS, with IGF-1 levels at 3 timepoints: before surgery $(n=9)$, following surgery $(n=9$; measured at $6-10$ weeks), and at latest follow-up ( $n=8$; mean 13 months postsurgery, range 4-28 months). $\mathrm{m}=$ months; $\mathrm{W}=$ weeks.

patients. ${ }^{11}$ Importantly, and in contrast to the current study, the operated cases comprised patients who had predominantly intrasellar disease remnants, with other treatment options (medical therapy and/or RT) preferred in those patients with clear cavernous sinus involvement.

PAs may invade adjacent structures, including the cavernous sinus, and this correlates with poor long-term remission rates. Microscopic dural invasion may be observed in up to $45.5 \%$ of PAs ${ }^{15}$ with higher rates observed in larger tumors. Postoperative recurrence rates of $8.8 \%$ were observed during long-term follow-up. ${ }^{15}$ The extent of parasellar disease visible on MRI may be a predictive marker for microscopic invasiveness, with extension beyond the intercarotid line and encasement of the ICA correlating with higher rates of invasiveness on histological examination. ${ }^{17}$ In our cohort of patients with parasellar disease, the potential risk of microscopic dural invasion raises the possibility that some patients will relapse eventually, despite achieving normal or near-normal biochemistry, which has been maintained to date during early follow-up (median 8 months, mean 13 months, range 4-28 months). Therefore, continued close surveillance will be necessary in this cohort. However, even if subsequent relapse occurs in a subgroup of these patients, given the rapid correction of $\mathrm{GH}$ and IGF-1 following revision surgery without the need for ongoing adjunctive medical therapy or RT (with follow-up $>2$ years in some instances), it could be reasoned that such revision surgery has delivered clear benefits for both the patient and the broader healthcare system. Reassuringly, no patients developed additional pituitary deficits or suffered other complications of what is inherently higher-risk surgery, but larger studies involving other centers will be required to confirm the safety of this approach.

From a surgical perspective, it is known that radiological evidence of parasellar extension does not necessarily mean that the cavernous sinus is invaded. Micko et al. ${ }^{17}$ have shown that the rates of true cavernous sinus invasion are $1.5 \%, 10 \%$, and $26.5 \%$ for Knosp grade 1, 2, and 3A adenomas, respectively. This rate goes up to $71 \%$ and $100 \%$ for grade $3 \mathrm{~B}$ and 4 tumors. Based on these findings, when there is postoperative "abnormal tissue" graded as Knosp 1, 2, or $3 \mathrm{~A}$ visible on MRI and the patient still has residual active acromegaly, it could be argued that surgical reexploration can be justified without the need for further imaging. Nevertheless, Met-PET/MR ${ }^{\mathrm{CR}}$ is a noninvasive technique that can clarify whether this parasellar "abnormal tissue" is or is not tumor and can therefore enable the surgeon to more accurately balance the benefits against the risks of revision surgery. In other words, with equivocal MRI appearances and parasellar disease, it is logical for surgeons-and patients - to be apprehensive about revision surgery; however, with confirmation by Met-PET/MR $\mathrm{MR}^{\mathrm{CR}}$ that one is dealing with tumor rather than scar tissue, the risk-benefit ratio of revision surgery becomes more favorable. We believe that in the era of precision medicine, this is important.

To confirm postoperative biochemical remission, we measured two variables, serum IGF-1 and glucose-suppressed serum GH, in line with international acromegaly clinical guidelines. ${ }^{8}$ In the surgical management of acromegaly, TSS can achieve IGF-1 normalization in up to $70.2 \%$ of all cases ${ }^{13}$ with parasellar disease extension and dural invasion strongly associated with poorer surgical 
outcomes. In our cohort, all 9 patients exhibited a rapid improvement in symptoms and abnormal biochemistry (Table 1), and, importantly, this has been maintained or improved further during longitudinal follow-up (Fig. 5), with all patients returning serum IGF-1 levels $\leq 1.2$ times ULN ( 6 of whom have achieved complete normalization) without further adjunctive therapy. In the 2 patients with persistent very mild elevations of serum IGF-1 at the last follow-up (cases 3 and 5), in the absence of any symptoms of active acromegaly and with glucose-suppressed GH values of $<0.20$ and $0.71 \mu \mathrm{g} / \mathrm{L}$, respectively, both have elected for surveillance follow-up at this time.

There is currently no literature that reports specifically on the surgical cure rates of repeat surgery for PA with a lateral sellar/parasellar remnant, partly reflecting the fact that such adenomas are commonly perceived as "inoperable." The pooled sensitivity and specificity of Met-PET/ $\mathrm{MR}^{\mathrm{CR}}$ may facilitate more accurate localization and therefore enable consideration of a surgical approach in more patients with these adenomas. The complexities in PET image interpretation, combined with an awareness of its limitations, indicate that the decision for surgical management should be guided by an experienced pituitary MDT, and that revision TSS should be undertaken by an experienced pituitary surgeon.

This study has several limitations. The sample size is small; therefore, while the study serves as an important proof of concept, larger cohorts of similar cases, managed across several centers, will be required to establish how molecular PET imaging might fit within modern algorithms for acromegaly management. An immediate challenge to answering this important question is the relatively short half-life (approximately 20 minutes) of the radiotracer carbon-11, which is generated using a particle accelerator, and adoption of this technique is therefore currently limited to centers with an on-site cyclotron. The development and adoption of alternative tracers (e.g., ${ }^{18} \mathrm{~F}-$ fluorinated) will likely be required for more widespread rollout of such services.

Although we did not encounter any complications of repeat surgery in our cohort, a more extended TS approach brings with it additional challenges and risks. Accordingly, it is logical to ask whether PET-guided stereotactic radiosurgery could represent a more attractive option for dealing with remnant lateral sellar/parasellar disease. However, it is important to take into account the latency period with respect to achieving biochemical remission following stereotactic radiosurgery, which can be longer than is often appreciated, with a recent systematic review estimating an overall mean biochemical remission of $44.3 \%$ at a mean follow-up of 59 months and a median time to remission at 41.5 months. Radiosurgery also carries with it a risk of inducing hypopituitarism. ${ }^{5}$

\section{Conclusions}

In summary, this study provides a proof of concept that Met-PET/MRCR has potential utility in the evaluation of lateral sellar/parasellar disease in patients with residual acromegaly following primary therapy and can guide targeted revision TSS.

\section{Acknowledgments}

This work was supported by the United Kingdom National Institute for Health Research Cambridge Biomedical Research Centre (W.A.B., R.S., O.K., D.G., H.C., J.M., I.M., M.G.) and by the Evelyn Trust, Cambridge.

\section{References}

1. Bashari WA, Senanayake R, Fernández-Pombo A, Gillett D, Koulouri O, Powlson AS, et al: Modern imaging of pituitary adenomas. Best Pract Res Clin Endocrinol Metab 33:101278, 2019

2. Bergström M, Muhr C, Lundberg POO, Långström B: PET as a tool in the clinical evaluation of pituitary adenomas. $\mathbf{J}$ Nucl Med 32:610-615, 1991

3. Elster AD: Modern imaging of the pituitary. Radiology 187:1-14, 1993

4. Feng Z, He D, Mao Z, Wang Z, Zhu Y, Zhang X, et al: Utility of 11C-methionine and 18F-FDG PET/CT in patients with functioning pituitary adenomas. Clin Nucl Med 41:e130e134, 2016

5. Gheorghiu ML: Updates in outcomes of stereotactic radiation therapy in acromegaly. Pituitary 20:154-168, 2017

6. Hwang J, Seol HJ, Nam DH, Lee JI, Lee MH, Kong DS: Therapeutic strategy for cavernous sinus-invading nonfunctioning pituitary adenomas based on the modified Knosp grading system. Brain Tumor Res Treat 4:63-69, 2016

7. Jane JA Jr, Starke RM, Elzoghby MA, Reames DL, Payne SC, Thorner MO, et al: Endoscopic transsphenoidal surgery for acromegaly: remission using modern criteria, complications, and predictors of outcome. J Clin Endocrinol Metab 96:2732-2740, 2011

8. Katznelson L, Laws ER Jr, Melmed S, Molitch ME, Murad MH, Utz A, et al: Acromegaly: an Endocrine Society clinical practice guideline. J Clin Endocrinol Metab 99:3933-3951, 2014

9. Knosp E, Steiner E, Kitz K, Matula C: Pituitary adenomas with invasion of the cavernous sinus space: a magnetic resonance imaging classification compared with surgical findings. Neurosurgery 33:610-618, 1993

10. Koulouri O, Hoole AC, English P, Allinson K, Antoun N, Cheow $\mathrm{H}$, et al: Localisation of an occult thyrotropinoma with ${ }^{11} \mathrm{C}$-methionine PET-CT before and after somatostatin analogue therapy. Lancet Diabetes Endocrinol 4:1050, 2016

11. Koulouri O, Kandasamy N, Hoole AC, Gillett D, Heard S, Powlson AS, et al: Successful treatment of residual pituitary adenoma in persistent acromegaly following localisation by 11C-methionine PET co-registered with MRI. Eur J Endocrinol 175:485-498, 2016

12. Koulouri O, Steuwe A, Gillett D, Hoole AC, Powlson AS, Donnelly NA, et al: A role for ${ }^{11} \mathrm{C}$-methionine PET imaging in ACTH-dependent Cushing's syndrome. Eur J Endocrinol 173:M107-M120, 2015

13. Kreutzer J, Vance ML, Lopes MB, Laws ER Jr: Surgical management of GH-secreting pituitary adenomas: an outcome study using modern remission criteria. J Clin Endocrinol Metab 86:4072-4077, 2001

14. Marko NF, LaSota E, Hamrahian AH, Weil RJ: Comparative effectiveness review of treatment options for pituitary microadenomas in acromegaly. J Neurosurg 117:522-538, 2012

15. Meij BP, Lopes M-BS, Ellegala DB, Alden TD, Laws ER Jr: The long-term significance of microscopic dural invasion in 354 patients with pituitary adenomas treated with transsphenoidal surgery. J Neurosurg 96:195-208, 2002

16. Melmed S: Acromegaly pathogenesis and treatment. J Clin Invest 119:3189-3202, 2009

17. Micko ASG, Wöhrer A, Wolfsberger S, Knosp E: Invasion of the cavernous sinus space in pituitary adenomas: endoscopic 
verification and its correlation with an MRI-based classification. J Neurosurg 122:803-811, 2015

18. Minniti G, Traish D, Ashley S, Gonsalves A, Brada M: Risk of second brain tumor after conservative surgery and radiotherapy for pituitary adenoma: update after an additional 10 years. J Clin Endocrinol Metab 90:800-804, 2005

19. Nomikos P, Buchfelder M, Fahlbusch R: The outcome of surgery in 668 patients with acromegaly using current criteria of biochemical 'cure'. Eur J Endocrinol 152:379-387, 2005

20. Rodriguez-Barcelo S, Gutierrez-Cardo A, Dominguez-Paez M, Medina-Imbroda J, Romero-Moreno L, Arraez-Sanchez $\mathrm{M}$ : Clinical usefulness of coregistered ${ }^{11} \mathrm{C}$-methionine positron emission tomography/3-T magnetic resonance imaging at the follow-up of acromegaly. World Neurosurg 82:468473, 2014

21. Taku N, Koulouri O, Scoffings D, Gurnell M, Burnet N: The use of ${ }^{11}$ carbon methionine positron emission tomography (PET) imaging to enhance radiotherapy planning in the treatment of a giant, invasive pituitary adenoma. BJR Case Rep 3:20160098, 2017

22. Tang BNT, Levivier M, Heureux M, Wikler D, Massager N, Devriendt $\mathrm{D}$, et al: ${ }^{11} \mathrm{C}$-methionine PET for the diagnosis and management of recurrent pituitary adenomas. Eur J Nucl Med Mol Imaging 33:169-178, 2006

23. Tritos NA, Mattsson AF, Vila G, Biller BMK, Klibanski A, Valluri S, et al: All-cause mortality in patients with acromegaly treated with pegvisomant: an ACROSTUDY analysis. Eur J Endocrinol 182:285-292, 2020

\section{Disclosures}

The authors report no conflict of interest concerning the materials or methods used in this study or the findings specified in this paper.

\section{Author Contributions}

Conception and design: Gurnell, Bashari, Gillett, Kolias. Acquisition of data: Bashari, Senanayake, Koulouri, Gillett, MacFarlane, Powlson, Fernandez-Pombo, Bano, Martin, Cheow, Mendichovszky, Tysome, Donnelly, Santarius, Kolias, Mannion. Analysis and interpretation of data: Bashari, Senanayake, Koulouri, Gillett, MacFarlane, Powlson, Fernandez-Pombo, Scoffings, Cheow. Drafting the article: Bashari, Senanayake, Koulouri, Gillett, Powlson. Critically revising the article: Gurnell, Bashari, Koulouri, Kolias. Reviewed submitted version of manuscript: all authors. Approved the final version of the manuscript on behalf of all authors: Gurnell. Administrative/ technical/material support: Bashari, Senanayake, Koulouri, Gillett, MacFarlane, Powlson, Fernandez-Pombo, Kolias. Study supervision: Gurnell.

\section{Correspondence}

Mark Gurnell: Wellcome Trust-MRC Institute of Metabolic Science, University of Cambridge, United Kingdom. mg299@ medschl.cam.ac.uk. 